\title{
Caractérisation des habitats benthiques et ponte des tortues marines autour du parc national de Campo-Ma'an (Cameroun)
}

\author{
I. AYISSI ${ }^{1 *}$, M. AKSISSOU ${ }^{1}$, M.TIWARI ${ }^{2}$ et J. FRETEY ${ }^{3}$ \\ ${ }^{1}$ Université Abdelmalek Essaâdi, Département de Biologie, Faculté de Sciences Tétouan 2121, Maroc. \\ ${ }^{2}$ NOAA-National Marine Fisheries Service, Southwest Fisheries Science Center, \\ 8604 La Jolla Shores Drive, La Jolla, California 92037, USA. \\ ${ }^{3}$ UICN-France, Muséum d'Histoire Naturelle, 36 rue Geoffroy Saint Hilaire, 75005 Paris, France. \\ ${ }^{*}$ Auteur correspondant ; E-mail: isidoreayissi@gmail.com
}

\section{RESUME}

Pour évaluer l'importance de la périphérie du parc national de Campo-Ma'an pour la création d'un parc marin, une caractérisation des habitats benthiques a été réalisée durant 24 mois (2008-2009) et de suivi des plages pour évaluer l'importance de la population dans ce site. L'étude s'est faite à travers des suivis saisonniers de collecte d'échantillons sous marins, grâce aux plongées sous marines dans les localités d'Ebodjé et de Mbodé. Des analyses révèlent 20 espèces d'algues marines, la distribution par groupes présente, 9 Rhodophycées (45\%), 6 Phéophycées (30\%) et 5 Clorophycées (25\%). Ces différentes espèces sont distribuées dans 11 familles: Cladophoraceae, Dictyotaceae et Chaetangiaceae avec 3 espèces chacune ; Caulerpaceae, Gelidiaceae et Gracilariaceae 2 espèces chacune; Scytosiphonaceae, Squamariaceae, Sargassaceae, Grateloupiaceae, et Rhodomelaceae avec une espèce chacune. Des analyses des contenus stomacaux des spécimens des tortues marines prises mortes au filet confirment que ces algues font parties de l'alimentation des espèces fréquentant la zone. Des patrouilles nocturnes sur les plages de ponte ont été menées d'octobre 2008 à Mars 2009 ainsi que des sorties diurnes dans les pêcheries au retour des pêcheurs pour l'identification des espèces présentes, le comptage des traces, les relevés biométriques. Des résultats nous révèlent un total de 3450 œufs provenant de 28 nids, soit une moyenne $150 \pm 21,6$ œufs. Les incubations présentent des durées de 60 à 80 jours avec une moyenne de 70 jours.

(c) 2013 International Formulae Group. All rights reserved.

Mots clés: Habitats benthiques, algues marines, ponte, tortues marines, Cameroun.

\section{INTRODUCTION}

La côte camerounaise est particulièrement riche en de nombreuses espèces fauniques et floristiques et particulièrement les tortues marines qui fréquentent ce littoral pour l'alimentation et la nidification (Ayissi, 2000 ; Angoni, 2005, 2010). La caractérisation de l'habitat des tortues marines et sa corrélation avec le régime alimentaire donne une image réaliste sur la zone de l'alimentation. Selon Fretey (1998), certaines espèces comme les femelles des tortues vertes qui nidifient dans les îles de l'Ascension, en plein milieu de l'Atlantique, vont jusqu'au niveau des côtes brésiliennes situées à plus de $2200 \mathrm{~km}$ de distance pour se nourrir. Les tortues luths se nourrissent de méduses, de tuniques et de crabes (Fretey, 
1998). Ainsi dans l'Atlantique Ouest, les tortues vertes se nourrissent des plantes marines telles que Thalassia testidinum et Syringodium filiforme. Les tortues olivâtres se nourrissent de crabes, tuniques et de petits invertébrés alors que les tortues imbriquées se nourrissent d'éponges, de tuniques, des mollusques et d'algues. La tortue verte est essentiellement herbivore, son alimentation varie suivant les océans et la distribution des espèces d'algues dans les fonds (Bjorndal, 1997).

Selon des travaux de suivi d'inventaires menés dans la zone de 1999 à 2002 par Angoni (2005), 294 tortues ont été baguées par des bagues Monel Eco et en majorité des vertes. Selon des données biométriques de ces espèces, la majorité est constituée des juvéniles ce qui pourrait renforcer l'hypothèse que cette zone est une aire alimentaire et par conséquent une zone de croissance des espèces (Angoni, 2005).

De ce fait, cette étude constitue l'évaluation de l'importance écologique de la zone de Mbendji/Lolabè pour l'alimentation et la reproduction des tortues marines, par conséquent fournir des informations indispensables de la zone en vue de la création d'un parc marin pour la protection des habitats, la conservation et la gestion durable des tortues marines.

\section{MATERIEL ET METHODES}

\section{Site d'étude}

Le site de notre étude est localisé dans les localités d'Ebodjé et de Mbodé à la périphérie du parc national de Campo-Ma'an, sur l'axe Kribi-Campo. Le climat de cette zone est du type équatorial type guinéen avec deux saisons sèches et deux saisons des pluies. La moyenne des précipitations annuelles est de $3000 \mathrm{~mm}$ de pluies par an et la température oscille autour de $25^{\circ}-28{ }^{\circ} \mathrm{C}$. La végétation de cette région appartient au grand ensemble de massif de forêt dense humide camerounaise de basse et moyenne altitude, dans le groupe de forêt littoral, constituée d'une végétation dense humide sempervirente de basse altitude à Sagoglottis gabonensis et Lophira alata, sous type biafréen. Les sols de cette région sont des sols jeunes sur roches métamorphiques et éruptives anciennes. La région de Campo-Ma'an est arrosée par de nombreux ruisseaux et rivières dont les plus importants sont Ngata, Likodo, Bikoka, Bititi, Bwanjo, Lobé, Ntem et Ebodjé qui se déversent tous dans la mer. La zone d'étude est présentée à la Figure 1.

La végétation de ces plages est très particulière par sa structure et sa composition. Il existe une strate arbustive située vers la limite supérieure des plages, on peut $\mathrm{y}$ reconnaître des espèces telles que Cocos nucifera, Carapa procera, sous les arbustes se développent des fourrées constituées d'herbes et d'espèces sablonneuses à l'instar de Hibiscus tiliaceus, Sporobolus virginicus, les Poacées du genre Panicum et Pennisetum, les Cypéracées telle que Cyperus articulans et les Fabacées. Les parties soumises à l'action des marées comportent des espèces à tiges traçantes émettant de nombreuses racines adventives pour s'agripper. Il s'agit notamment d'Ipomoea mauritiana, Cynedon sp., Euphorbia glaucophylla, les plages méridionales rocheuses abritent de nombreuses bryophytes aquatiques associées à des microphanérophytes constituant ainsi des zones privilégiées d'alimentation de plusieurs organismes vivants.

Selon Folack et al. (1999), Atangana (1996), Morin et Kuete (1989) ces plages connaissent une action anthropique persistante à cause de leur envahissement anarchique par des grands travaux, des habitations individuelles, la présence de nombreux pêcheurs et des plantations sur ces plages. 


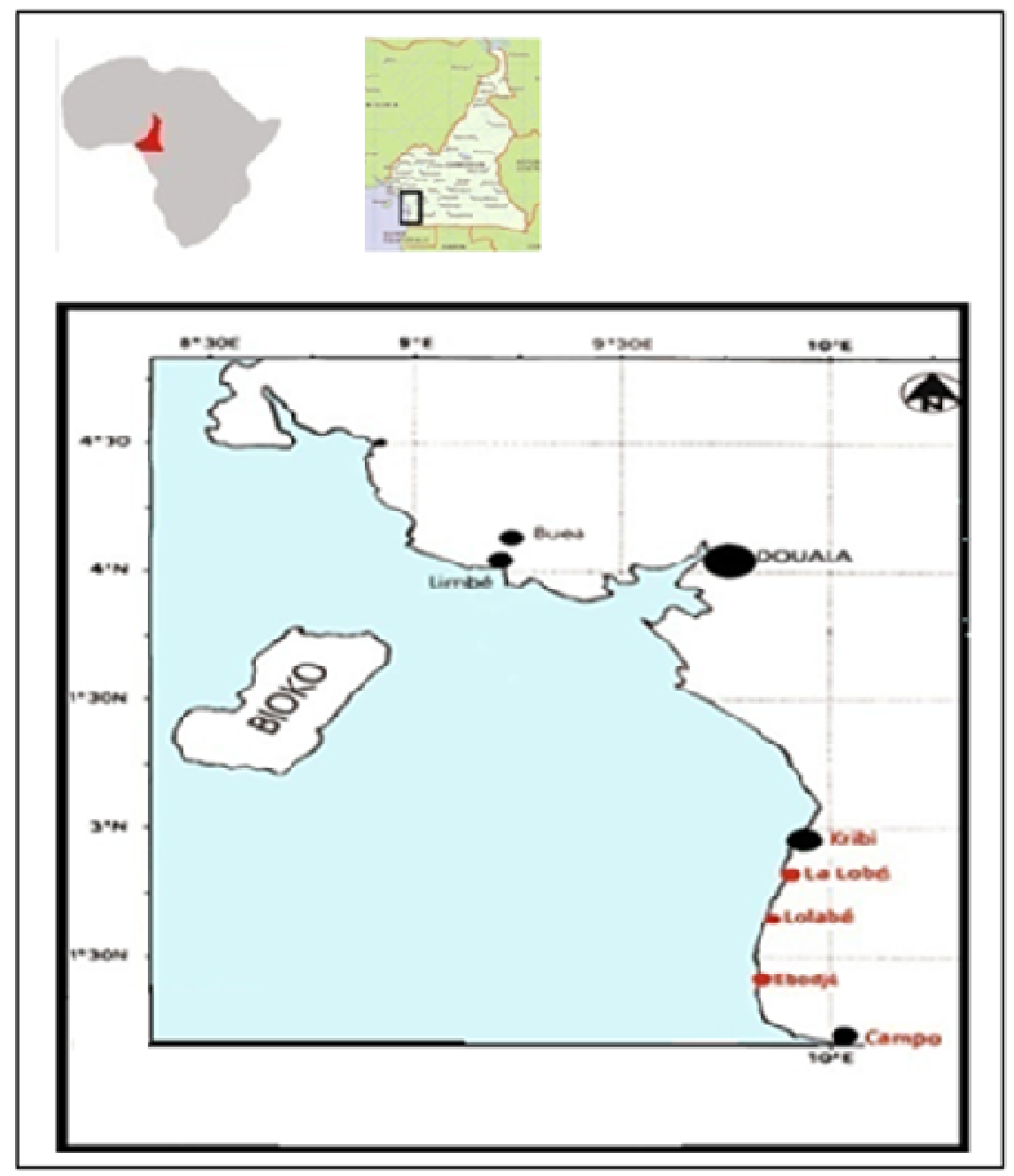

Figure 1 : Carte de la zone d'étude (Source : Projet Kudu Cameroun).

\section{Collecte des données}

Les 9 principales plages de ponte de Mbendji à Békolobè ont été explorées quotidiennement par deux patrouilles nocturnes pendant 6 mois d'octobre 2008 à mars 2009 soit quatre passages par site en fonction des marées montantes et hautes afin de déterminer le nombre de femelles montées pour pondre, d'identifier les individus et évaluer les nids.

Lors de ces patrouilles, nous nous servions de la Clé de détermination des tortues marines de l'Atlantique (Fretey, 1999) pour identifier les individus observés. A chaque observation, certains éléments particuliers sont décrits comme :

- La biométrie par la longueur et la largeur courbe dossière grâce à un mètre ruban pour estimer l'âge des individus observés ;

Les signes particuliers, pour déterminer le nombre de vertébrales, de costales, de préfrontales pour identifier des aberrations d'écaillure et les comportements bizarres ;

- La pose d'une bague MONEL ECO est placée à l'aide des pinces fortes sur l'une des 
pattes antérieures pour le marquage des individus.

Pour l'évaluation des aires alimentaires, des plongées ont été effectuées par un guide local qui était chargé de récolter des échantillons de fonds marins qui ont été immergés dans une solution d'eau de mer et du formol à $10 \%$ o pendant $5 \mathrm{mn}$ et essorées avant prises de vues pour l'identification au laboratoire mais certaines identification ont été faites sur le terrain à l'aide des guides d'identification de terrain.

Par ailleurs des contenus stomacaux de certaines tortues vertes mortes, capturées accidentellement par les filets des pêcheurs ont été identifiés grâce à un guide d'identification selon Diane et al. (1989). et confirmation par un expert algologue au Maroc afin de voir si ces espèces d'algues sont réellement celles observées au niveau des herbiers.

\section{Analyse}

Les différentes données collectées sont traitées et saisies dans les logiciels Excel 2007. Le traitement de ces informations et la synthèse des données ont permis de réaliser des tableaux de contingence dans un format adapté, puis exportées pour une suite d'analyses et de traitements adéquats. Pour les différentes analyses nous avons établies l'estimation des fréquences de la population de chaque espèce par rapport à l'effectif total selon la formule :

Fréquence $=\frac{x i}{\Sigma x i} \times 100$

$-\mathrm{x}_{\mathrm{i}}$ : Nombre d'individus de l'espèce ;

$-\sum x i:$ Effectif total d'individus.

\section{RESULTATS}

\section{Caractérisation des herbiers sous-marins}

Pour la composition des aires alimentaires de cette zone, 20 espèces d'algues marines ont été répertoriées (Figure 2). Celles-ci sont indiquées dans la check-list du Tableau 1.
$\mathrm{Au}$ vue de ces résultats, les rhodophycées (Chaetangiaceae, Gelidiaceae, Gracilariaceae, Grateloupiaceae, Squamariaceae et Rhodemelaceae) sont les plus représentées avec 6 familles, suivies des phéophycées avec 3 familles (Dictyotaceae, Sargassaceae et Scytosiphonaceae) et enfin Deschlorophyceae avec 2 familles (Caulerpacea et Cladophoraceae).

Pour les paramètres écologiques de l'environnement marin de ce milieu, le substrat du sol présente un caractère rocheuxsablonneux et le niveau de la mer variant de 1 à $3 \mathrm{~m}$, avec une moyenne $1,76 \mathrm{~m}$.

La température de l'air est permanemment élevée et varie entre $25{ }^{\circ} \mathrm{C}$ à $30{ }^{\circ} \mathrm{C}$ avec une moyenne de $28,89{ }^{\circ} \mathrm{C}$. L'eau de mer est claire en saison sèche et turbide en saison des pluies lors des apports continentaux des fleuves et rivières côtiers.

\section{Evaluation de la composition des contenus stomacaux}

Les observations des contenus stomacaux des tortues prises accidentellement au filet nous révèlent que la majorité des algues identifiées dans la zone d'étude se retrouvent dans l'alimentation de ces espèces. Si les chlorophycées sont difficiles à identifier au niveau des échantillons, l'observation macroscopique des rhodophycées et phéophycées est très palpable. Les ordres identifiés sont ceux des Dictyotales, Fucales, Nemaliales, Gelidiales, Gigartinales et Ceramiales. Toutefois l'identification des espèces présentes reste limitée car la majorité des contenus obtenus étant à un état de digestion avancée, ceci pourrait aussi constituer la limite d'identification chlorophycées malgré leur forte présence dans ces habitats benthiques.

Evaluation de la population des tortues marines et leur statut de nidification

Des données des tortues observées et baguées au cours des saisons 2007, 2008 et 
2009 présentent une distribution spécifique des fréquences (Figure 3).

Le suivi journalier de 15 kilomètres (de Mbendji à Bekolobe) a présenté 63 traces symétriques des tortues olivâtres Lepidochelys olivacea. au cours de la saison 2008-2009, 54 nids ont été recensés tandis que 9 tortues sont reparties dans la mer sans pondre des œufs dans les localités de Mbendji $(n=4)$, Likodo $(\mathrm{n}=1)$, Beyo $(\mathrm{n}=3) ; 7$ nids ont été pillés de leurs œufs par les populations riveraines et 28 nids ont été transplantés dans l'enclos situé à Ebodjé pour un suivi de leur incubation (Figure 4).

Un nombre total de 3450 œufs provenant de 28 nids pour une moyenne de $150 \pm 21,6$ œufs par ponte ont été transplantés. Au total 2252 bébés tortues ont été libérés de l'écloserie, pour une durée d'incubation variant de 60 à 80 jours pour une moyenne de 70 jours. Le taux de réussite des incubations a été de 98,85\%. Ces œufs provenaient essentiellement des plages d'Ebodjé, de Mbendji, Ebodjé, Likodo et Ipenyendjé. Au total 33 tortues ont été marquées à la bague Monel et 7 nids seulement sur 75 ont été braconnés ceci grâce aux actions de sensibilisation et l'implication des pêcheurs dans les activités de suivi.

La tortue Lepidochelys olivacea est la seule espèce venue pondre sur les plages du parc national de Campo Ma'an pendant la campagne 2008/2009 (Figure 4). Les pontes de cette espèce sont constantes entre les mois d'octobre et novembre pour chuter brusquement au cours des mois de février et de mars avec un pic en décembre et janvier.

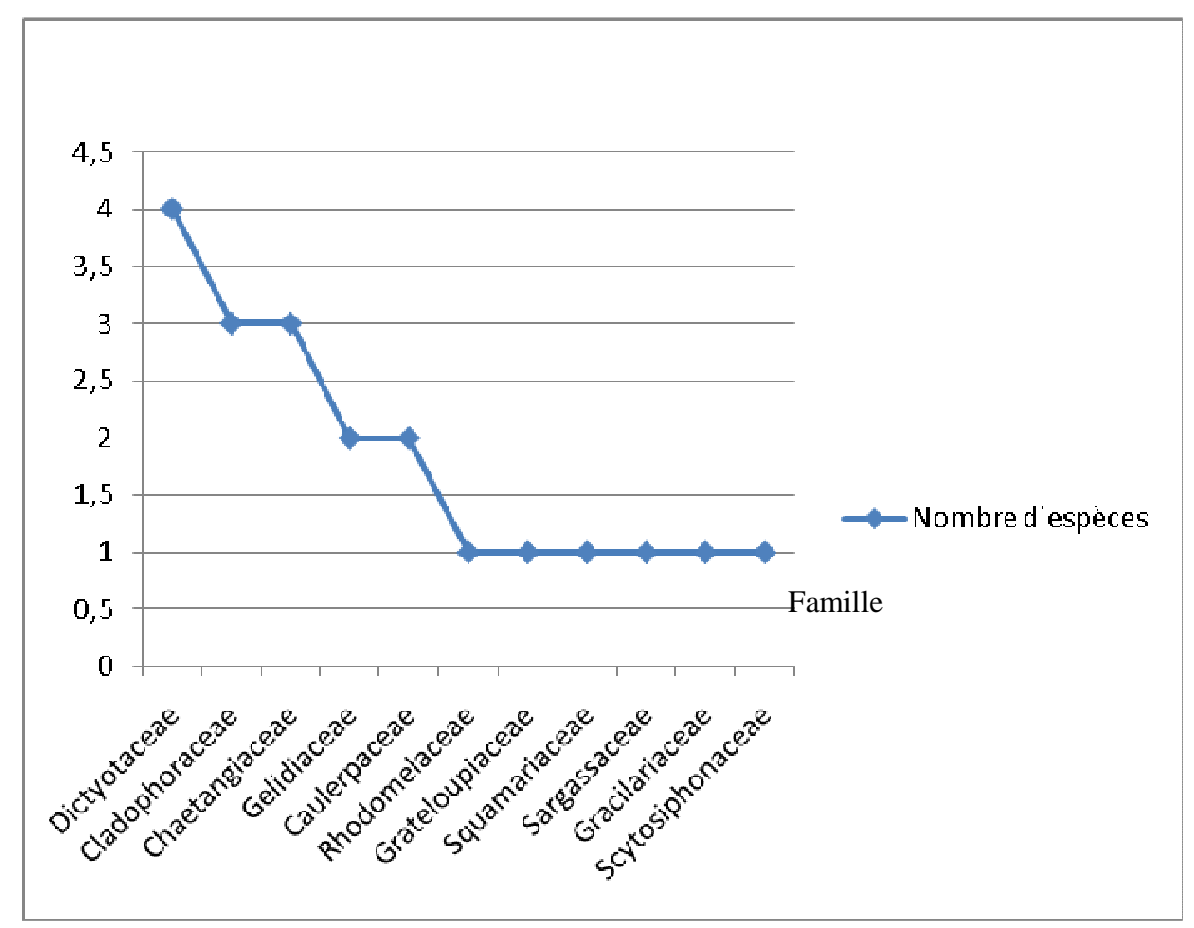

Figure 2: Distribution de fréquence des familles d'algues marines en fonction du nombre d'espèces des années 2008 et 2009. 


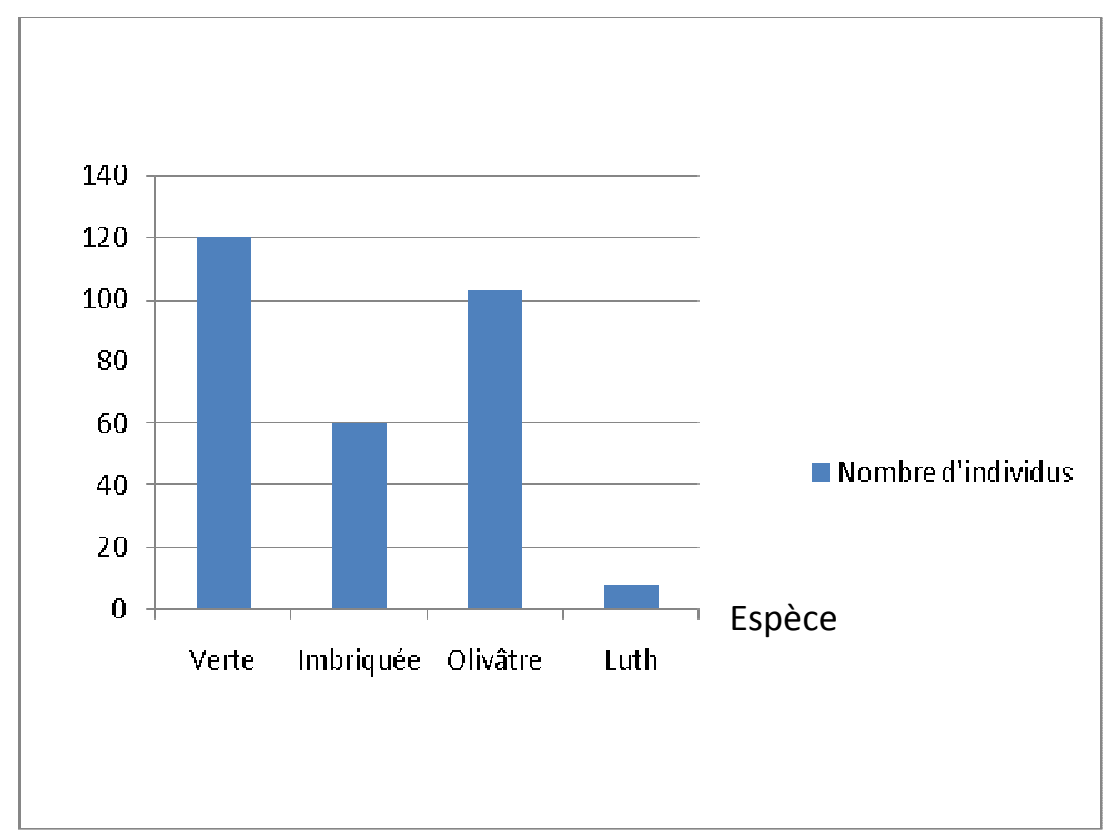

Figure 3: Distribution de fréquence du nombre de tortues observées en fonction des espèces dans la zone durant la période 2007- 2009.

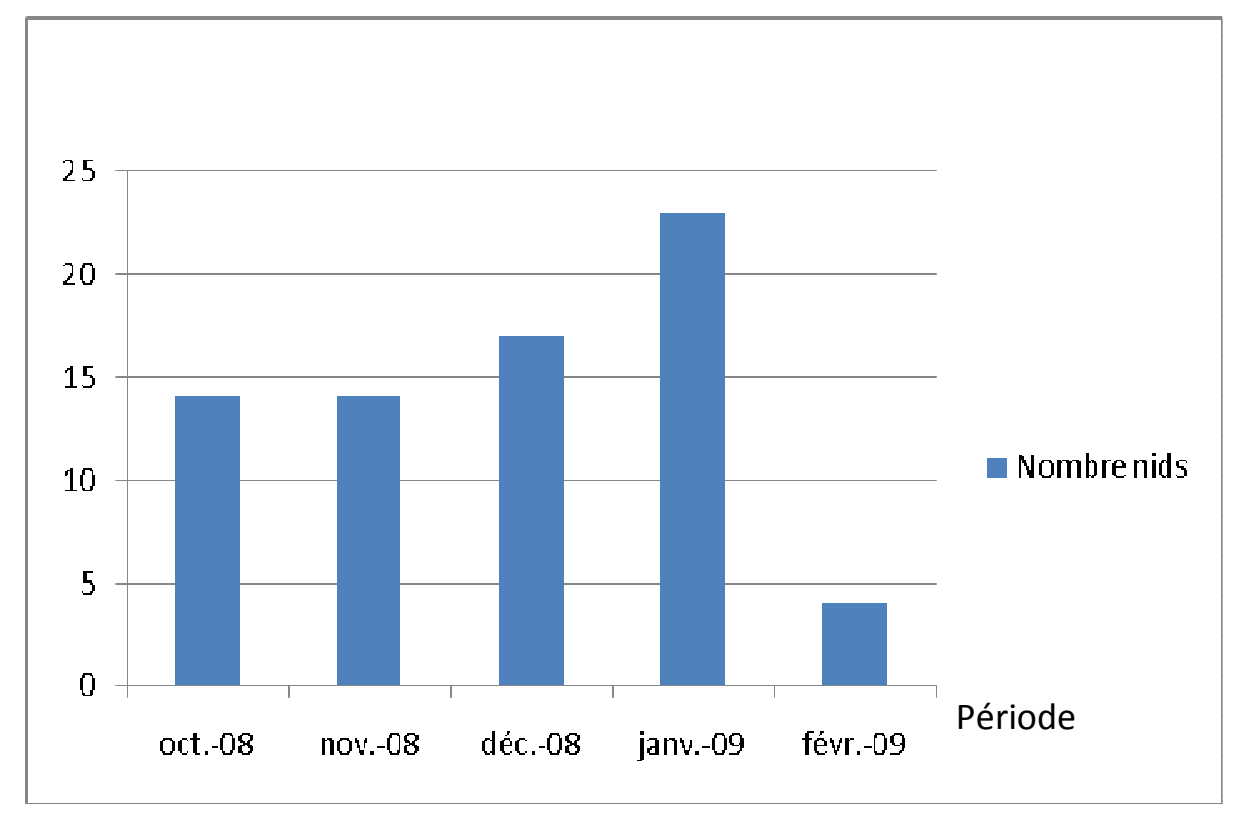

Figure 4: Distribution de fréquence des nids de pontes de Lepidochelys olivacea au cours de la campagne 2008-2009. 
Tableau 1 : Liste des espèces d'algues observées au cours des années 2008 et 2009.

\begin{tabular}{lccc}
\hline Espèces & Groupes & Ordres & Familles \\
\hline Caulerpa racemosa & Chlorophycées & Caulerpales & Caulerpaceae \\
C. sp & Chlorophycées & Caulerpales & Caulerpaceae \\
Chaetomorpha crassa & Chlorophycées & Cladophorales & Cladophoraceae \\
C. linum & Chlorophycées & Cladophorales & Cladophoraceae \\
C. aerea & Chlorophycées & Cladophorales & Cladophoraceae \\
Padina gymnosperma & Phéophycées & Dictyotales & Dictyotaceae \\
P.ulva & Phéophycées & Dictyotales & Dictyotaceae \\
Dictyota dichotoma & Phéophycées & Dictyotales & Dictyotaceae \\
D. mertensii & Phéophycées & Dictyotales & Dictyotaceae \\
Sargassum sp & Phéophycées & Fucales & Sargassaceae \\
Hydroclathus clathratus & Phéophycées & Scytosiphonales & Scytosiphonaceae \\
Galaxaura marginata & Rhodophycées & Nemaliales & Chaetangiaceae \\
G. oblongata & Rhodophycées & Nemaliales & Chaetangiaceae \\
G. subverticillata & Rhodophycées & Nemaliales & Chaetangiaceae \\
Gelidilium pusilium & Rhodophycées & Gelidiales & Gelidiaceae \\
G. nigrescens & Rhodophycées & Gelidiales & Gelidiaceae \\
Gracilaria cervicornis & Rhodophycées & Gigartinales & Gracilariaceae \\
Halymenia duchassaignii & Rhodophycées & Cryptonemiales & Grateloupiaceae \\
Peyssonnelia sp & Rhodophycées & Cryptonemiales & Squamariaceae \\
Laurencia obtusa & Rhodophycées & Ceramiales & Rhodemelaceae \\
\hline
\end{tabular}

\section{DISCUSSION}

Le nombre d'espèces d'algues est presque égal à celui observé par Jouan (2002) qui décrit 22 espèces d'Algues répertoriées dans la zone sur substrat rocheux. Mais ces espèces sont différentes malgré que les mêmes familles soient représentées. Cette différence serait dû que ses observations ont été faites de manière systématique dans la zone sur une dizaine de kilomètres alors nos travaux se sont focalisés sur deux transects distincts de $1 \mathrm{~km}$ chacun. Par ailleurs ces algues ont été retrouvées dans les contenus stomacaux des jeunes tortues vertes ce qui confirme l'hypothèse que cette zone serait une zone d'alimentation des tortues marines.

La distribution des espèces prises au filet et baguées dans la zone présente une fréquence plus élevée pour la verte et l'olivâtre. Si cette zone est une aire de nidification pour l'olivâtre, tel n'est pas le cas pour la verte et l'imbriquée ceci pourrait confirmer que cette zone est une aire d'alimentation pour ces espèces et par conséquent une aire de croissance pour ces dernières. Ces résultats confirment ceux obtenus par Fretey (2001) et Angoni (2005, 2010) car les espèces lues sont la verte et l'olivâtre.

Les résultats de ponte contrastent un peu avec les résultats des années précédentes années qui indiquaient aussi les pontes de Dermochelys coriacea dans cette zone (Fretey, 2001); ceci pourrait être dû aux phénomènes du repos biologique ou à la diminution de la population de cette espèce.

\section{Conclusion}

Les principaux résultats révèlent que 20 espèces de plantes marines ont été répertoriées et réparties en 11 familles. Certains spécimens de ces plantes marines ont été retrouvés dans les contenus stomacaux des tortues. Par ailleurs le suivi des plages de ponte confirme la nidification de la tortue olivâtre car l'espèce étant caractéristique de 
ces zones de ponte avec 3450 œufs provenant de 28 nids, soit une moyenne $150 \pm 21,6$ œufs. Les incubations présentent des durées de 60 à 80 jours avec une moyenne de 70 jours.

Ainsi la périphérie du parc national de Campo-Ma'an est une zone de prédilection pour l'alimentation des tortues vertes car de nombreuses observations des espèces font état des juvéniles vertes et imbriquées qui fréquentent essentiellement la zone pour leur alimentation confirme des informations d'Ayissi (2000), Ayissi et al. (2006a, 2006b, 2007) et Angoni (2005, 2010).

Compte tenue de l'importance de cette zone pour l'alimentation des tortues marines des mesures d'aménagement seraient la bienvenue comme : Par ailleurs l'approfondissement des activités de recherche pour établir des mesures de compensation en harmonie avec le développement de la pêche, l'exploration pétrolière et la construction du port en eau profonde de Kribi qui sont tous des projets en cours dans la zone ;

Son classement en aire de protection marine pour réduire la pression des impacts anthropiques sur ce site sensible pour la biodiversité marine.

\section{REFERENCES}

Angoni H. 2005. Biologie et Ecologie des Tortues Marines en Rapport avec les Ecosystèmes côtiers. Conservation et Aménagement. Thèse de Doctorat $3^{\text {è }}$ Cycle. Université de Yaoundé I, p. 125.

Angoni H, Amougou A, Bilong BCF, Fretey J. 2010. La tortue marine au Cameroun, genre Lepidochelys: nidification, biométrie de Lepidochelys olivacea (Eschscholtz, 1829) (Reptilia, Cheloniidae) dans la réserve de faune de Campo (Sud Cameroun). Int. J. Biol. Chem. Sci., 4(3): 649-656.

Atangana ER. 1996. Biogéographie des écosystèmes côtiers et marins. Rapport Plan National de Gestion de l'Environnement, p. 34.
Ayissi I. 2000. Projet d'un Ecotourisme <<Tortues Marines>> dans la Région d'Ebodjé. (Unité Technique Opérationnelle de Campo-Ma'an). Mémoire DESS, Université de Yaoundé I, p. 57.

Ayissi I, Ajonina GN, Usongo L, Fretey J. 2006. Etude Préliminaire des tortues marines dans la Réserve de Faune de Douala-Edéa (Cameroun) en vue de l'établissement d'une stratégie de conservation. In Proceedings. $2^{\mathrm{e}}$ Congrès International sur la Conservation des Chéloniens: 195-198, Saly-Sénégal, Mai 2003.

Ayissi I, Angoni H, Amougou A, Fretey J. 2006. Ecotourism and Natural Resources (Case study of sea turtles in CampoMa'an National Park in Cameroon).

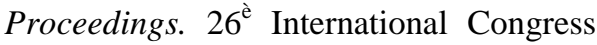
on sea turtles: 211-212, Creta-Greece, June 2006.

Ayissi I, Angoni H, Amougou A, Fretey J. 2007. Impacts of Artisanal fishing on natural resources case study on sea turtles along Cameroon coastline (West-Africa).

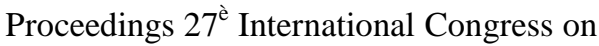
sea turtles in Myrtle Beach (SouthCarolina, USA).

Bjorndal KM. 1997. Foraging ecology and nutrition of sea turtles. In The Biology of Sea Turtles (vol 1), Lutz PL, Musick JA (eds). CRC Press: Boca Raton, FL; 199231.

Boye M, Baltzer F, Caratini C. 1974. Mangrove of the Wouri estuary. Int. Symp. Of biology and management of mangrove, Honolulu, 435-455.

Carr A. 1957. Notes on the Zoogeography of Atlantic Sea Turtles of the Genus Lepidochelys. Rev. Biol. Trop., 5(1): 4561.

Diane SL, Mark ML, Katina EB, James NN. 1989. Marine Plants of the Caribbean. A Field Guide from Florida to Brazil. Smithsonian Institution Press: Washington, DC. 
Folack J, Mbome IL, Bokwe A, Tagang A. 1999. Cameroon Coastal Profile, p. 102.

Fretey J. 1998a. Quelques Notions sur l'identification et la Biologie des tortues marines. Les différentes espèces. Atelier Guadeloupéen sur les tortues marines. p. 93.

Fretey J. 1998b. Statut des tortues marines en Afrique de l'Ouest - Afrique Centrale. 5. Le Cameroun. Suivi scientifique et conservation. Rapport UICN / ECOFAC, p. 32.

Fretey J. 1998c. Marine Turtles of the Atlantic Coast of Africa - Tortues Marines de la Façade Atlantique de l'Afrique. UNEP / CMS Techn. Publ. 1. p. 254.

Fretey J. 1999. Fretey J. 1999. Suivi et conservation des tortues marines dans la réserve de Campo-Ma'an. Rapport du projet Campo-Ma'an. 40 p.

Fretey J. 2001. Biogeography and Conservation of Marine Turtles of the Atlantic Coast of Africa / Biogéographie et Conservation des Tortues Marines de la Côte Atlantique de l'Afrique. CMS Technical Series Publication, UNEP/CMS: Bonn, Germany ; 429.
Jouan D. 2002. Rapport de Recherche, Projet d'Aménagement et de Conservation de la Biodiversité de Campo-Ma'an, p 26.

Kramkimel JM, Bousquet B. 1987. Mangrove d'Afrique et de Madagascar: Les Mangroves du Cameroun. CEE, SECA: Luxembourg; 127-137.

Loveridge W. 1997. Revision of the African Tortoises and Turtles of the Suborder Cryptodira. Bull. Mus. Comp. Zool., 115(6): 163-557.

Morin K M. 1989. Le Littoral Camerounais: problèmes morphologiques. Trav. Labo. Goegr. Phys. appliquée. Inst. Geogr. Univ. Bordeaux III, vol.11; pp 5-53.

Sayer JA, Harcourt CS, Collins NM. 1992. The Conservation Atlas of Tropical Forest Africa. Macmillan Publishing Ltd: London.

Spelleberg IF. 1982. Biology of Reptiles. An Ecological Approach. Chapman \& Hall: New-York; p.158.

Tornier. 1902. Die Crocodile, Schildkroten und Eidechsen in Kamerun. Zool Jalrb., 15: 163-677. 